\title{
A Study on LINEX Loss Function with Different Estimating Methods
}

\author{
N. Khatun, M. A. Matin \\ Department of Statistics, Jahangirnagar University, Savar, Dhaka, Bangladesh \\ Email: nasrin@juniv.edu, matinju@yahoo.com
}

How to cite this paper: Khatun, N. and Matin, M.A. (2020) A Study on LINEX Loss Function with Different Estimating Methods. Open Journal of Statistics, 10, 52-63.

https://doi.org/10.4236/ojs.2020.101004

Received: December 30, 2019

Accepted: January 16, 2020

Published: January 19, 2020

Copyright (อ 2020 by author(s) and Scientific Research Publishing Inc. This work is licensed under the Creative Commons Attribution International License (CC BY 4.0).

http://creativecommons.org/licenses/by/4.0/

(c) (i) Open Access

Abstract
LINEX means linear exponential loss function which used in the analysis of
statistical estimation and prediction problem which rises exponentially on
one side of zero and almost linearly on the other side of zero. It is used in
both overestimation and underestimation problems. Ali Shadrokh and Has-
san Pazira [1] presented Shrinkage estimator in Gamma Type-II Censored
Data under LINEX loss function. In that paper, they have explained how the
LINEX loss function works however no practical or detail explanations were
given in terms of changing the shape parameter and the error function. In
this study we have explained how the LINEX loss function works through
practical or detail explanations in terms of changing the shape parameter and
the error function, also see how the loss function works with the data gener-
ated from gamma distribution through resampling methods to compare the
performance of LINEX loss function considering the relative estimation error
and usual estimation error through generating random numbers from gam-
ma distribution like randomization method and by using bootstrapping sam-
ples. The very intention is to find out which resampling method performs
well in using the LINEX loss function. Using Monte Carlo Simulations these
estimators are compared. It is doing draw random number from the gamma
distribution and finds the maximum likelihood estimate of $\theta$ is $\frac{\sum_{i=1}^{n} x_{i}}{2 n}$ and using this estimator to explain the LINEX loss function

$L(\Delta)=\left(\mathrm{e}^{c \Delta}-c \Delta-1\right) ; \quad c \neq 0, \Delta=\left(\frac{\hat{\theta}}{\theta}-1\right)$ or $\Delta=\hat{\theta}-\theta$, where $c$ is the shape parameter and $\hat{\theta}$ is any estimate of the parameter $\theta$. The shape of this loss function is determined by the value of $c$. In the analysis we use the values of shape parameter $c=-0.25,-0.50,-0.75,-1$ and $c=0.25,0.50,0.75$, 1. The same procedure is done by using bootstrapping method, and finally compared between this two methods. The relative estimation error should be 
used instead of the estimation error where the LINEX loss function works better in both of the cases. Between the two estimators, bootstrap method is better work because although the characteristics are same, bootstrap method is more dispersed than others.

\section{Keywords}

Gamma Distribution, LINEX Loss Function, Bootstrap Method, Estimation Error, Relative Estimation Error

\section{Introduction}

In some estimation and prediction problems, the use of a symmetric loss function is appropriate. However, a given positive error may be more serious than a given negative error of the same magnitude or vice versa. Ali et al. [1] presented shrinkage testimators for Gamma Type II censored data under the invariant version of the LINEX loss function. They explained how the LINEX loss function worked, but there were no details or practical explanations given on how the LINEX loss function works in changing the shape parameter and the error function. This study is initiated to see how the LINEX loss function works with the data generated from Gamma distribution by using resampling methods such as Bootstrapping to compare the results in Ali et al. [1]. Andreou et al. [2] considered the problem of model uncertainty in forecasting volatility using forecast combinations and a flexible family of asymmetric loss functions that allow the possibility that an investor would attach different preferences to a high vis-a-vis low volatility period. Despite the flexibility of the LINEX loss function for the estimation of a location parameter, it appears not to be suitable for the estimation of scale parameters and other quantities. For these reasons Basu and Ibrahimi [3] defined a modified LINEX loss function. Christoffersen et al. [4] used linex loss function in a study of optimal point prediction where different asymmetric loss functions are tested. Bayes and frequentist estimators for two parameters Weibull with uncensored observations under survival and hazard functions used in life testing and survival analysis are considered (Guure et al. [5]). On the other hand, when the underestimation is more serious than the overestimation or vice-versa then an asymmetric loss function should be used. For two parameter exponential families it is difficult to estimate the parameters when both are unknown. To obtain an optimum decision for two parameter exponential families a two-parameter conjugate utility function is used. In this case, optimum decision for one parameter exponential family under conjugate utility function is obtained and thereafter extends the results to the two-parameter exponential family. To have optimum decisions, for example, for normal, trinomial and inverse Gaussian distribution parameters under two-parameter conjugate utility function are used. Jasim [6] derived Bayes estimator for the scale parameter $\theta$ in Gamma distribution when lamda is known and equals to 2 . The estimator 
is obtained under the squared error and LINEX loss function and comparison of risks are made. Pandey and Malik [7] proposed an improved estimator for the variance in exponential distribution and studied the properties under the LINEX loss function. Patton and Timmermann [8] considered the evaluation of forecast optimality in economics and finance has almost exclusively been conducted under the assumption of mean squared error loss. The optimal forecast should be unbiased and forecast errors should be serially uncorrelated at single period horizon with increasing variance as the forecast horizon grows. Analytically they have shown that the standard properties of optimal forecasts can be invalid under asymmetric loss and non-linear data generating processes in turn may be very misleading as a benchmark for an optimal forecast. In a practical situation, Sengupta [9] obtained that the asymmetric loss functions are preferred over squared error loss functions as the former is more appropriate than the latter in many estimation problems. The problem of fixed precision point estimation of a linear parametric function in betas for a multiple linear regression model using asymmetric loss function is considered. Due to the presence of nuisance parameters the sample size for the estimation problem is not known before and hence the recourse of adaptive multistage sampling methodologies is considered. Singh [10] suggested an improved estimator for the mean using the LINEX loss function that dominates the estimator under the same loss function. The mathematical expression of the improved estimator of fourth power of mean and an improved estimator for the common mean in negative exponential distribution are obtained under the LINEX loss function Singh [11] proposed a pooled estimator for the common variance in normal distribution to study the properties under the LINEX loss function. Singh et al. [12] obtained the Bayes' estimator of the Generalized-Exponential scale and shape parameters using Lindley's approximation under asymmetric loss function. The proposed estimators have been compared with the corresponding MLE for their risks based on simulated samples from the Generalized-Exponential distribution. LINEX loss function rises approximately exponentially on one side of zero and approximately linearly on the other side (Soliman et al., [13]). Varian [14] found that an asymmetric linear loss function also rises exponentially on the one side of the zero and rises linearly on the other side of the zero.

\section{Materials and Methods}

Ali et al. presented shrinkage testimators for Gamma Type II censored data under the invariant version of the LINEX loss function. They have explained how the function works however no practical details or explanations were given in terms of changing the shape parameter and the error function used. In this regard, the present study initiated the idea to see how the loss function works with the generated gamma distribution through resampling methods like randomization method and bootstrapping method. The very intention is to find out which resampling method performs well in using the LINEX loss function. 
The distribution mentioned is the widely used two parameter gamma distribution inherently related with the exponential family distribution. The density of two parameter Gamma distribution is given by $f(x ; \lambda, \theta)=\frac{x^{\lambda-1}}{\sqrt{(\lambda)} \theta^{\lambda}} \mathrm{e}^{\frac{-x}{\theta}} ; x \geq 0, \lambda, \theta \geq 0$. In order to use the two resampling methods to generate data let fix the scale parameter $\theta=1$ and shape parameter $\lambda=2$ for the two parameter gamma distribution. Draw a random sample of size 100 repeating 100 times to have maximum likelihood estimate of $\theta$ which can be used to explain the LINEX loss function. This is very much the matter of randomization method. For bootstrapping draw a random sample of size 100 there after using the first sample to draw 100 samples of the same size with replacement to have the bootstrap estimate of $\theta$ which can be used to explain the LINEX loss function. Consider the LINEX loss function $L(\Delta)=\left(\mathrm{e}^{c \Delta}-c \Delta-1\right)$ where the shape parameter $c \neq 0$ and $\Delta=\left(\frac{\hat{\theta}}{\theta}-1\right)$ where $(\hat{\theta}-\theta)$ is the pitman difference and $\hat{\theta}$ is any estimate of the parameter $\theta$. It rises approximately exponentially on one side of zero and approximately linearly on the other side. It is convex while its shape is determined by the value of $c$. It is identified a family of loss functions $L(\Delta)$ where $\Delta$ is either the estimation error $(\hat{\theta}-\theta)$ or the relative estimation error $(\hat{\theta}-\theta) / \theta$. Such a loss function is useful whenever the actual losses are nonnegative, increases with estimation error, the overestimation is more (less) serious than the underestimation of the same magnitude and losses increase at a faster (slower) rate with the overestimation error. Note that $T_{n}=\hat{\theta}$ is a statistic which is an estimator of $\theta$, $\Delta=T_{n}-\theta$ is the error, $\mathrm{c}$ is the shape parameter. In this study, the values $\{c=$ $(-0.25,-0.50,-0.75,-1) ; c=(0.25,0.50,0.75,1)\}$ of the shape parameter are used.

\section{Results and Discussion}

In order to understand well the behavior of the LINEX loss function a numerical illustration with real life data and simulated data from two parameter gamma distribution with varieties of scale parameter with the other parameter constant. In first place, a real life numerical data of rainfall of Dhaka station, Bangladesh for the month of January from 1968 to 2013 is considered. Draw a random sample of size 100 repeating 100 times to have maximum likelihood estimate of $\theta$ which can be used to explain the LINEX loss function. This is very much the matter of randomization method. For bootstrapping draw a random sample of size 100 there after using the first sample to draw 100 samples of the same size with replacement to have the bootstrap estimate of $\theta$ which can be used to explain the LINEX loss function. Generate 100 random number from gamma distribution with the scale parameter $\theta=1$. The maximum likelihood estimate of $\theta$ is $\hat{\theta}=\frac{\sum_{i=1}^{n} x_{i}}{2 n}=8.218002$. 
In this phase, the comparison between estimation error and relative estimation error is made for the LINEX loss function considering the real life data.

\subsection{LINEX Loss Function Using Relative Estimation Error:}

$$
\text { Consider the Relative Estimation Error as } \Delta=\left(\frac{\hat{\theta}}{\theta}-1\right)
$$

The negative value of $c$ gives more weight to overestimation that reflects the asymmetry (Figure 1(a)). For large positive values of the shape parameter reflects the asymmetry (Figure 1(b)). For small values of $|c|$ the LINEX loss function is asymmetric (Figure $1(\mathrm{c})$ ). For large values of $|c|$ the LINEX loss function is almost asymmetric (Figure 1(d)). For $c<0$, it rises almost linearly when the estimation error is $(\hat{\theta}-\theta)>0$ (Figure $1(\mathrm{e})$ ) and it rises almost exponentially when the estimation error is $(\hat{\theta}-\theta)<0$ Figure $1(\mathrm{f}))$.

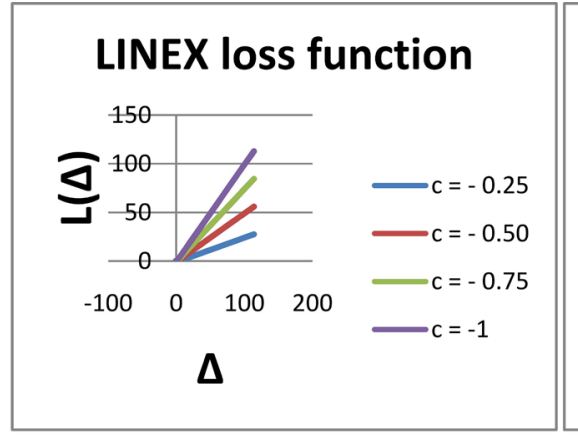

(a): with $c<0$

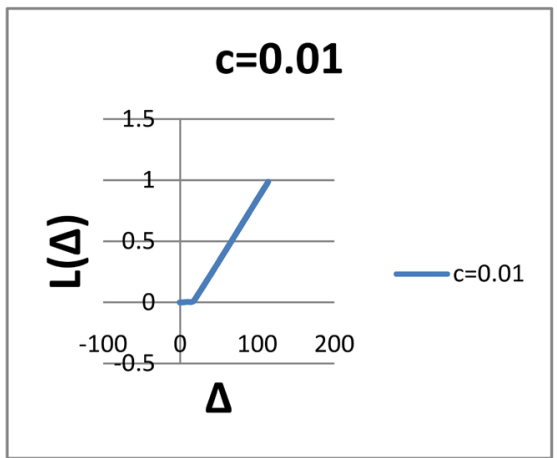

(c): with $c=0.01$

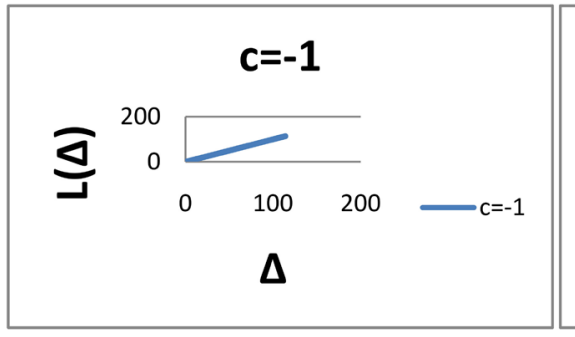

(e): with $c=-1$ and $(\hat{\theta}-\theta)>0$

\section{LINEX loss function}

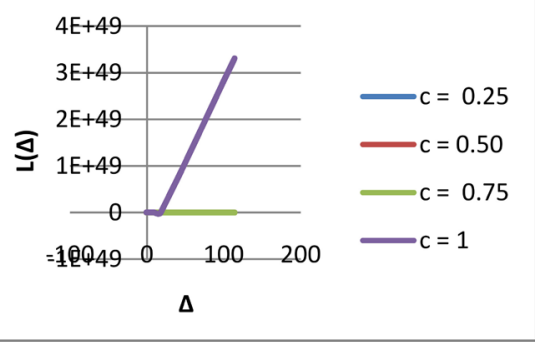

(b): with $c>0$

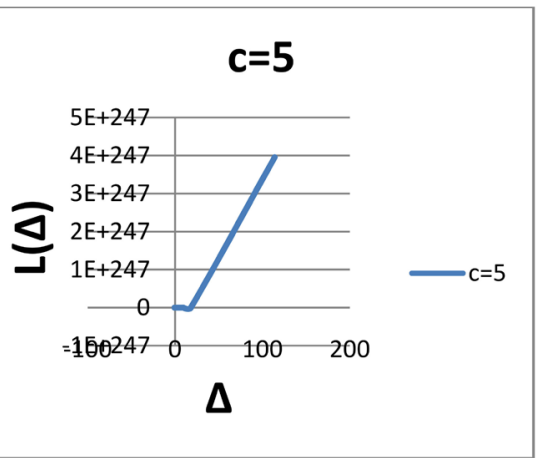

(d): with $c=5$

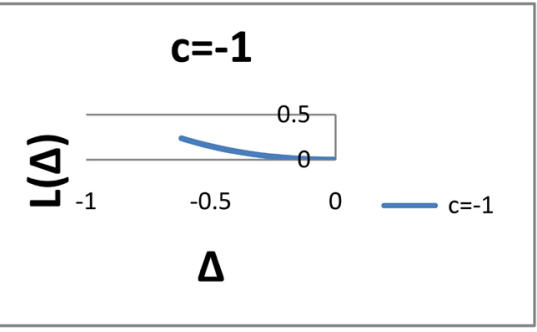

(f): with $c=-1$ and $(\hat{\theta}-\theta)<0$

Figure 1. Linex loss functions considering relative estimation error. 


\subsection{LINEX Loss Function Using Estimation Error: Consider the}

$$
\text { Estimation Error as } \Delta=(\hat{\theta}-\theta)
$$

The negative value of $c$ provides more weight to underestimation compared to the overestimation that reflects the almost symmetry (Figure 2(a)). For large positive values of the shape parameter reflects the degree of symmetry (Figure 2(b)). For small values of $|c|$ the LINEX loss function is almost symmetric (Figure 2(c)). For large values of $|c|$ the LINEX loss function is almost symmetric (Figure $2(\mathrm{~d})$ ). For $c<0$, it rises almost linearly when the estimation error is $(\hat{\theta}-\theta)>0 \quad$ (Figure $2(\mathrm{e}))$ and almost exponentially when the estimation error is $(\hat{\theta}-\theta)<0 \quad$ (Figure 2(f)).

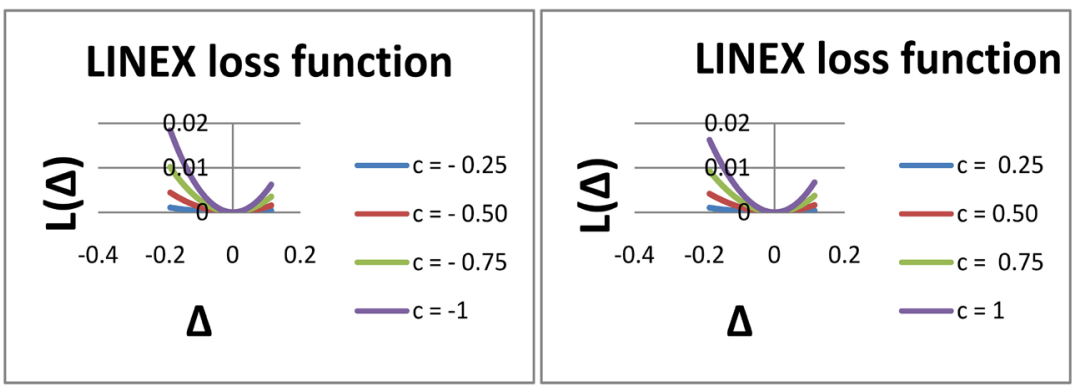

(a): with $c<0$

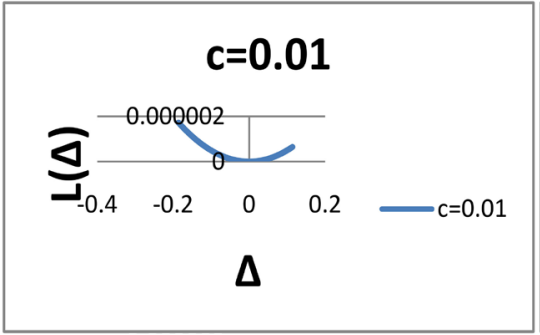

(c): when $c=0.01$

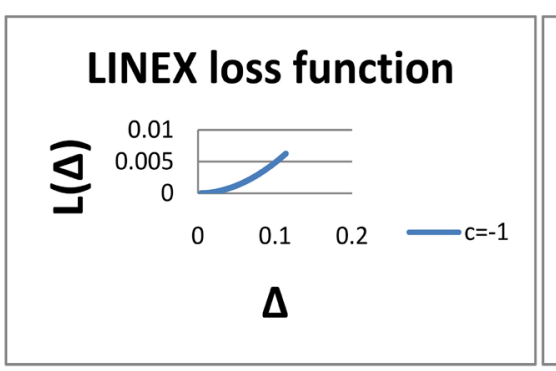

(e): with $c=-1$ and $(\hat{\theta}-\theta)>0$ (b): with $c>0$

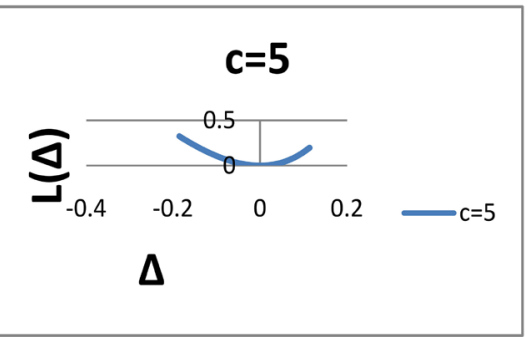

(d): with $c=5$

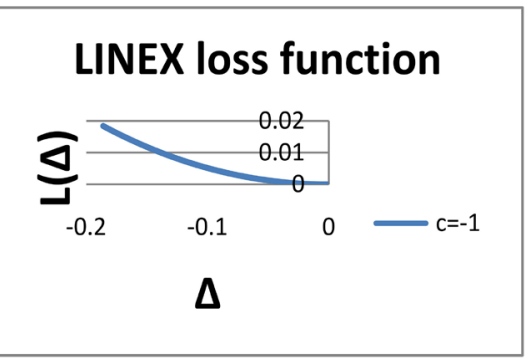

(f): with $c=-1$ and $(\hat{\theta}-\theta)<0$

Figure 2. Linex loss functions considering estimation error.

\subsection{LINEX Loss Function Using Relative Estimation Error:}

Consider the Relative Estimation Error as $\Delta=\left(\frac{\hat{\theta}}{\theta}-1\right)$

The negative value of $c$ gives more weight to overestimation that reflects asym- 
metry (Figure 3(a)). The positive value of $c$ gives more weight to overestimation for $c=1$ that reflects the degree of asymmetry (Figure 3(b)). For small values of $|c|$ the LINEX loss function is almost asymmetric (Figure 3(c)). For large values of $|c|$ the LINEX loss function is almost asymmetric (Figure $3(d)$ ). For $c<0$, it rises almost linearly when the estimation error is $\Delta=\left(\frac{\hat{\theta}}{\theta}-1\right)>0 \quad$ (Figure $3(\mathrm{e})$ ) and almost exponentially when the estimation error is $\Delta=\left(\frac{\hat{\theta}}{\theta}-1\right)<0 \quad$ (Figure 3(f)).

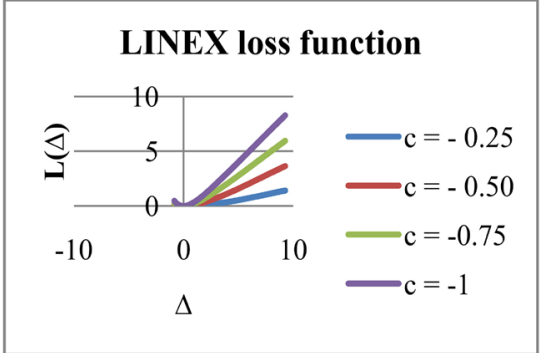

(a): with $c<0$

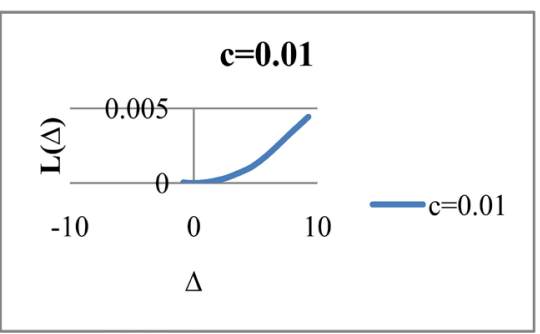

(c): with $c=0.01$

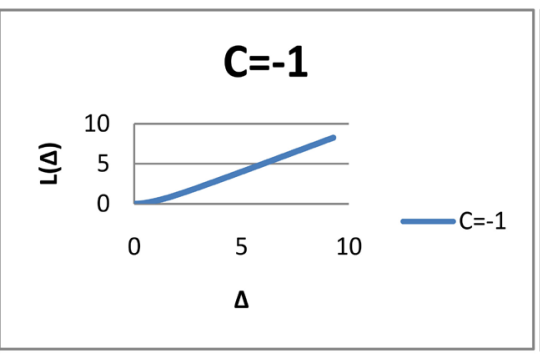

(e): with $c=-1$ and $\Delta=\left(\frac{\hat{\theta}}{\theta}-1\right)>0$

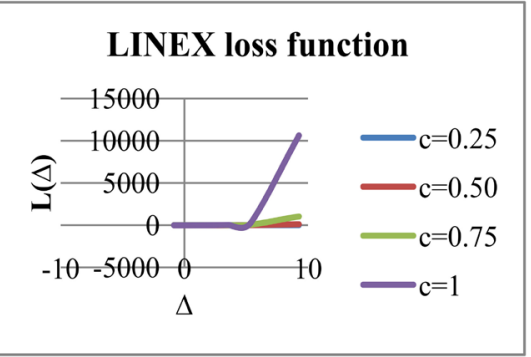

(b): with $c>0$

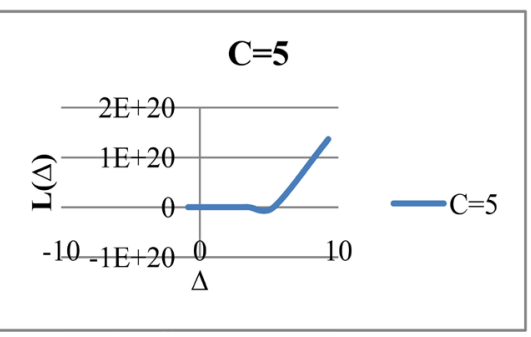

(d): with $c=5$

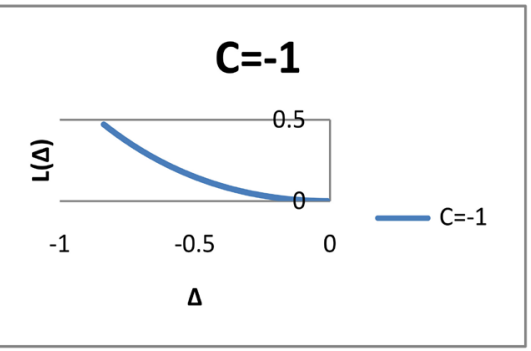

(f): with $c=-1$ and $\Delta=\left(\frac{\hat{\theta}}{\theta}-1\right)<0$

Figure 3. Linex loss function considering relative estimation error.

\subsection{LINEX Loss Function Using Estimation Error: Consider the Estimation Error as $\Delta=(\hat{\theta}-\theta)$}

The negative value of $c$ provides more weight to underestimation whose magnitude reflects the degree of asymmetry (Figure 4(a)). The positive value of $c$ does not provide more weight to overestimation whose magnitude reflects the degree 
of asymmetry (Figure $4(\mathrm{~b})$ ). For small values of $|c|$ the LINEX loss function is almost symmetric but not far from squared error loss function (Figure 4(c)). For large values of $|c|$ the LINEX loss function is almost asymmetric (Figure $4(\mathrm{~d})$ ). For $c<0$, it rises almost linearly when the estimation error is $\Delta=\left(\frac{\hat{\theta}}{\theta}-1\right)>0$ (Figure $\left.4(\mathrm{e})\right)$ and it rises almost exponentially when the estimation error is $\Delta=\left(\frac{\hat{\theta}}{\theta}-1\right)<0$ (Figure $4(\mathrm{f})$ ).

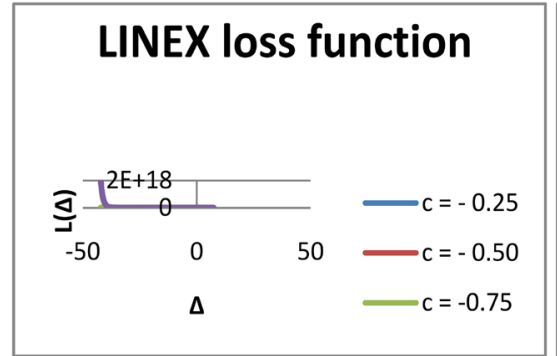

(a): with $c<0$

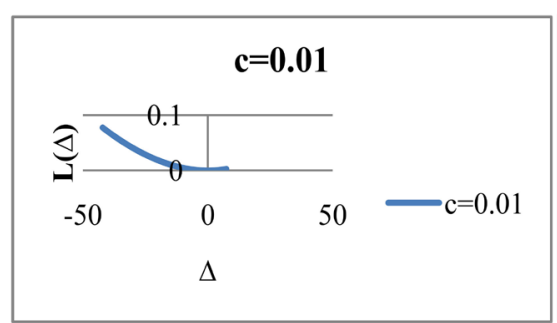

(c): with $c=0.01$

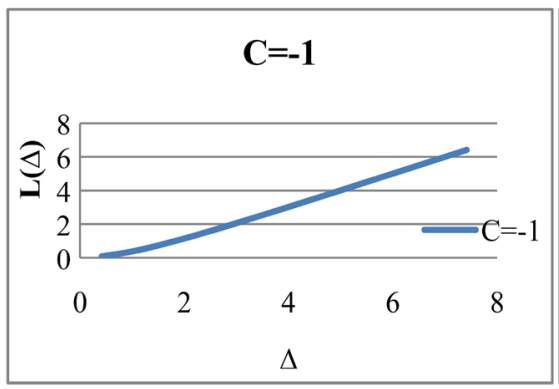

(e): with $c=-1$ and $(\hat{\theta}-\theta)>0$

\section{LINEX loss function}

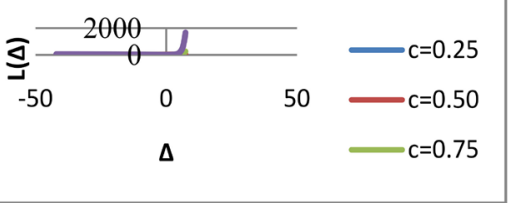

(b): with $c>0$

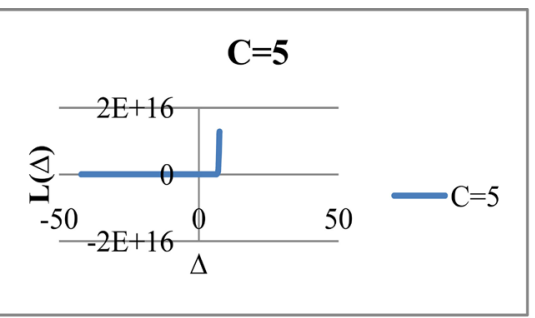

(d): with $c=5$

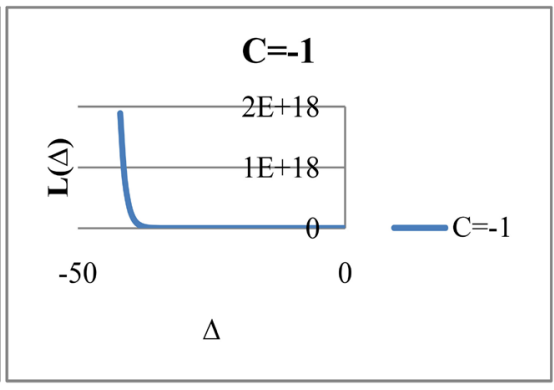

(f): with $c=-1$ and $(\hat{\theta}-\theta)<0$

Figure 4. Linex loss function considering estimation error.

\subsection{LINEX Loss Function Using Bootstrapping}

In second place, simulated data from two parameter gamma distribution with scale parameter $\theta=1$ is considered. Samples are drawn using bootstrapping method to generate $n=100$ bootstrap samples of size 100 each. The bootstrap estimate of $\theta$ is found to be $\hat{\theta}=15.9214$. In this phase, the comparison between estimation error and relative estimation error is made for the LINEX loss function considering the simulated bootstrapping data. 
3.5.1. LINEX Loss Function Using Relative Estimation Error: Consider the Relative Estimation Error as $\Delta=\frac{\hat{\theta}}{\theta}-1$

The negative value of $c$ gives more weight to overestimation that reflects asymmetry (Figure 5(a)). The positive value of $c$ gives more weight to overestimation whose magnitude reflects the degree of asymmetry (Figure 5(b)). For small values of $|c|$ the LINEX loss function is almost asymmetric and for large values of $|c|$ the LINEX loss function is almost asymmetric (Figure 5(c), Figure 5(d)). For $c<0$, it rises almost linearly when the estimation error is $(\hat{\theta}-\theta)>0$ and it rises almost exponentially when the estimation error is $(\hat{\theta}-\theta)<0$ (Figure 5(e), Figure 5(f)).

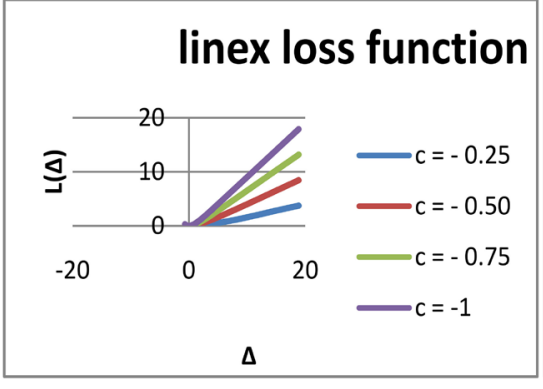

(a): with $c<0$

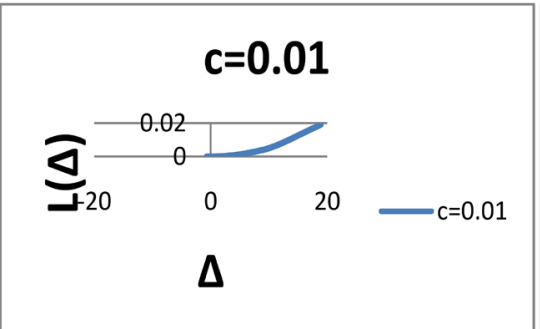

(c): with $c=0.01$

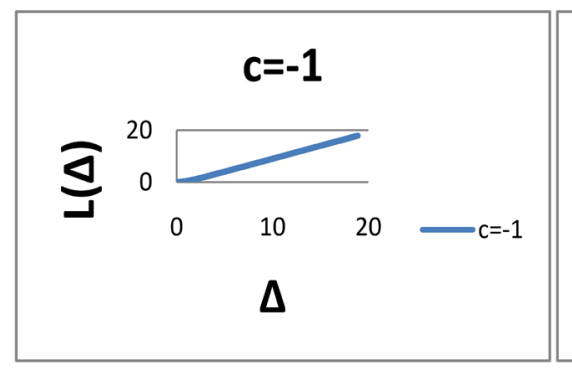

(e): with $c=-1$ and $(\hat{\theta}-\theta)>0$

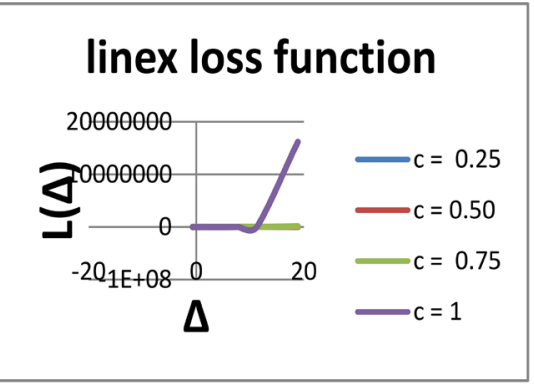

(b): with $c>0$

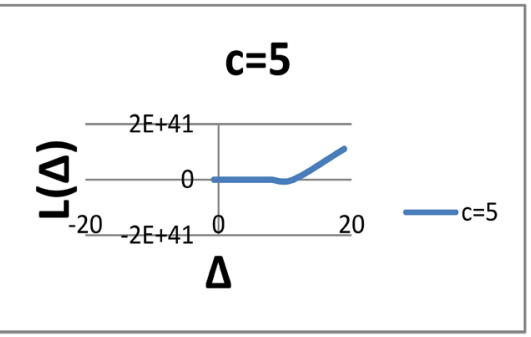

(d): with $c=5$

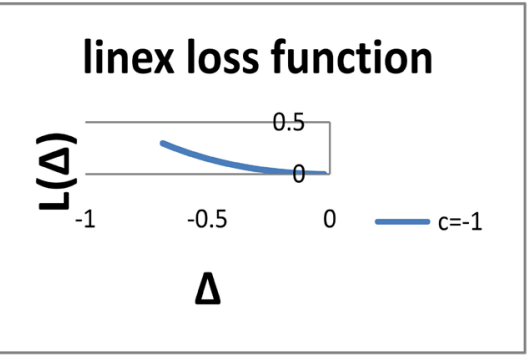

(f): with $c=-1$ and $(\hat{\theta}-\theta)<0$

Figure 5. Linex loss function considering relative estimation error.

\subsubsection{LINEX Loss Function Using Estimation Error: Consider the Estimation Error as $\Delta=(\hat{\theta}-\theta)$}

The negative value of $c$ gives more weight to underestimation that reflects the asymmetry (Figure 6(a)). The positive value of $c$ gives more weight to overestimation whose magnitude reflects the asymmetry (Figure 6(b)). For small values 
of $|c|$ the LINEX loss function is almost symmetric and for large values of $|c|$ the LINEX loss function is almost asymmetric (Figure 6(c), Figure 6(d)). For $c<0$, it rises almost linearly when the estimation error is $(\hat{\theta}-\theta)>0$ and it rises almost exponentially when the estimation error is $(\hat{\theta}-\theta)<0$ (Figure 6(e), Figure 6(f)).

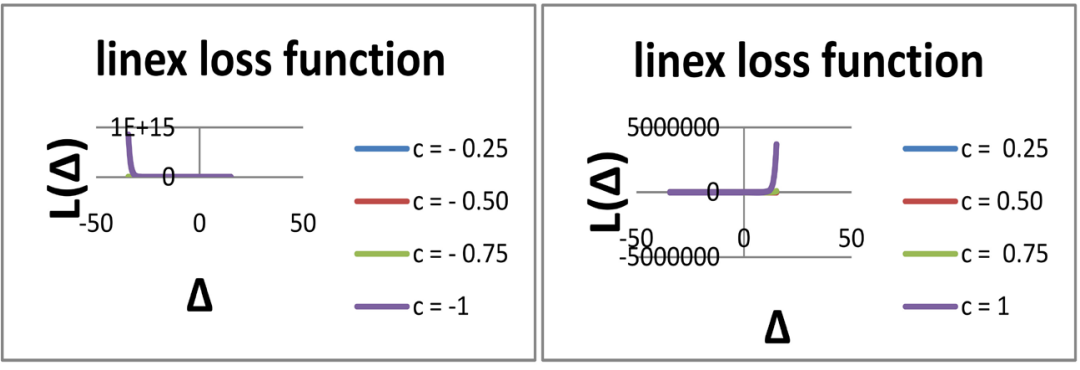

(a): with $c<0$

(b): with $c>0$

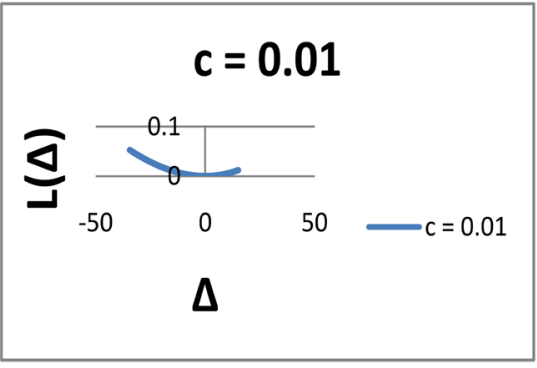

(c): with $c=0.01$

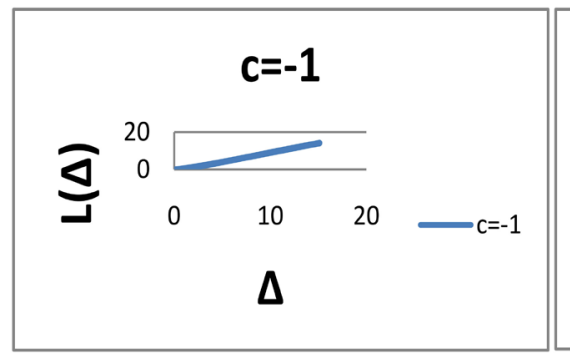

(e): with $c=-1$ and $(\hat{\theta}-\theta)>0$

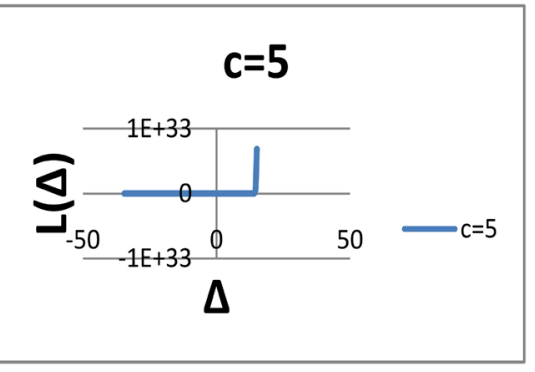

(d): with $c=5$
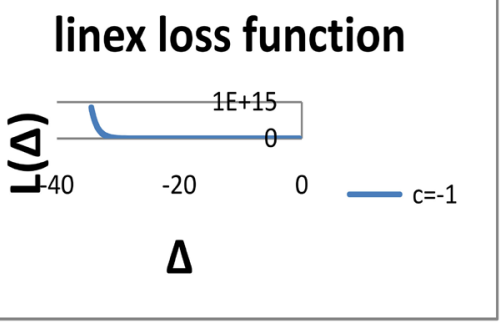

(f): with $c=-1$ and $(\hat{\theta}-\theta)<0$

Figure 6. Linex loss function considering estimation error.

\section{Conclusions}

It is concluded that using relative estimation error, the LINEX loss function, for the negative values of the shape parameter, gives more weight to overestimation in showing that the distribution is asymmetric while for the positive values it gives weight to overestimation in showing that the distribution is asymmetric too. So, for the positive values of the shape parameter, the condition of the LINEX loss function is satisfied. On the other hand, using the estimation error for negative values of the shape parameter, it gives more weight to underestimation in showing that the distribution is asymmetric while for the positive values of $c$ it gives more weight to overestimation that reflects the asymmetry. It may be 
concluded that for positive values of the shape parameter, the condition of the LINEX loss function is satisfied. So, the estimation error rather than the relative estimation error works better in the application of the LINEX loss function.

In using repeated samples, for the negative values of the shape parameter, the LINEX loss gives more weight to overestimation showing that the distribution is asymmetric. For positive values of $c$ it gives more weight to overestimation that reflects the degree of asymmetry. It may be concluded that for positive values of the shape parameter, the condition of the LINEX loss function is satisfied however, it is more spreader compared to the original random sample. On the other hand, using estimation error for negative values of the shape parameter gives weight to underestimation in showing that the distribution is asymmetric. So, the condition of the LINEX loss function is satisfied. For positive values of $\mathrm{c}$ it gives more weight to overestimation that reflects the degree of asymmetry. So, for positive values of the shape parameter, the condition of the LINEX loss function is satisfied however, it is more widely compared to the original random sample. In this case, it is also seen that estimation error rather than the relative estimation error works better in the application of the LINEX loss function.

It is thus concluded that the estimation error should be used instead of the relative estimation error where the LINEX loss function works better. Out of the two estimators, the bootstrapping performed better compared to the randomization methods since under the same characteristics bootstrap method is more spreader than the others. All the conditions of the LINEX loss function have been fulfilled in both the cases but in each of the characteristics of the LINEX loss function bootstrapping is outperformed.

\section{Acknowledgements}

At first, all praises are due to Allah, for helping me to complete my thesis. I want to express my sincere gratitude to my supervisor, Professor Dr. M. A. Matin, who introduced me to the LINEX loss function. It is an opportunity for me to acknowledge my gratitude to my honorable teacher Professor Dr. M. A. Matin, Department of Statistics, Jahangirnagar University, Savar, Dhaka, Bangladesh. $\mathrm{He}$ is an ideal as well as one of the most respectable teachers of Statistics Department, choice to the topics of my thesis. He spent his valuable time providing his advice, guidance and encouragement in order to complete my thesis.

\section{Conflicts of Interest}

The authors declare no conflicts of interest regarding the publication of this paper.

\section{References}

[1] Ali, S. and Pazira, H. (2013) Shrinkage Testimator in Gamma Type II Censored Data under LINEX Loss Function. Open Journal of Statistics, 3, 245-257. https://doi.org/10.4236/ojs.2013.34028

[2] Andreou, E., Kourouyiannis, C. and Kourtellos, A. (2012) Volatility Forecast Com- 
binations Using Asymmetric Loss Functions.

[3] Basu, A.P. and Ebrahimi, N. (1991) Bayesian Approach to Life Testing and Reliability Estimation Using Asymmetric Loss Function. Journal of Statistical Planning and Inferences, 29, 21-31. https://doi.org/10.1016/0378-3758(92)90118-C

[4] Christoffersen, P.F. and Diebold, F.X. (1997) Optimal Prediction under Asymmetric Loss. Economic Theory, 13, 808-817. https://doi.org/10.1017/S0266466600006277

[5] Guure, C.B., Ibrahim, N.A., Adam, M.B., Bosomprah, S. and Ahmed, Al.O.M. (2010) Bayesian Parameter and Reliability Estimate of Weibull Failure Time Distribution.

[6] Jasim, W.A.L. (2010) Bayes Estimator of One Parameter Gamma Distribution under Quadratic and LINEX Loss Function. Iraqi Journal of Statistical Science, 16, 13-28.

[7] Pandey, B.N. (1997) Testimator of the Scale Parameter of the Exponential Distribution Using LINEX Loss Function. Communication in Statistics, Theory and Methods, 26, 2191-2202. https://doi.org/10.1080/03610929708832041

[8] Patton, A.J. and Timmermann, A. (2007) Properties of Optimal Forecasts under Asymmetric Loss and Nonlinearity. Journal of Econometrics, 140, 884-918. https://doi.org/10.1016/j.jeconom.2006.07.018

[9] Sengupta, R.N. (2008) Use of Asymmetric Loss Functions in Sequential Estimation Problem for the Multiple Linear Regression. Journal of Applied Statistics, 35, 245-261. https://doi.org/10.1080/02664760701833388

[10] Singh, B.K. (2013) Estimation of Mean and Its Function Using Asymmetric Loss Function. International Journal of Soft Computing, Mathematics and Control (IJSCMC), 2, 27-44.

[11] Singh, B.K. (2016) Estimation of Common Variance in Normal Distribution Using Linex Loss Function. Columbia International Publishing Journal of Applied Mathematics and Statistics, 3, 59-79. https://doi.org/10.7726/jams.2016.1005

[12] Singh, R., Singh, S.K., Singh, U. and Singh, G.P. (2008) Bayes Estimator of Generalized Exponential Parameters under Linex Loss Function Using Lindleys Approximation. Data Science Journal, 7, 65-75. https://doi.org/10.2481/dsj.7.65

[13] Soliman, A.A. (2000) Comparison of Linex and Quadratic-Bayes Estimators Foe the Rayleigh Distribution. Communication in Statistics-Theory and Methods, 29, 95-107. https://doi.org/10.1080/03610920008832471

[14] Varian, H.R. (1975) A Bayesian Approach to Real Estate Assessment. In: Savage, L.J., Feinberg, S.E. and Zellner, A., Eds., Studies in Bayesian Econometrics and Statistics. In Honor of L. J. Savage, North-Holland Pub. Co., 195-208. 